\title{
Nuclear Gas Turbine Propulsion System for a Long Endurance Titan Aerial Vehicle
}

\author{
Luke S. Colby ${ }^{*}$ \\ Georgia Institute of Technology, Guggenheim School of Aerospace Engineering, Atlanta, GA, 30332 \\ Robert Braun ${ }^{\dagger}$ and Ravi Prakash \\ Georgia Institute of Technology, Guggenheim School of Aerospace Engineering, Atlanta, GA, 30332
}

\begin{abstract}
An innovative propulsion system concept that enables powered flight on Saturn's largest moon, Titan, is discussed. This propulsion system concept uses waste heat from a NASA Multi-Mission Radioisotopic Thermoelectric Generator (MMRTG) to power a gas turbine engine. The propulsion system captures MMRTG waste heat by utilizing the in-situ resources of Titan's cold dense nitrogen atmosphere as a working fluid, passed through a heat exchanger. The heated gas is then run through a turbine to extract electrical power significantly greater than that available from the MMRTG's thermoelectric effect. In addition to analysis, an experimental system was constructed to validate the feasibility of the proposed concept. This investigation compares the results obtained with this experimental system to analytic predictions. Experimental system performance exceeding 500 watts of measured power output was achieved. This propulsive performance enables consideration of a robotic vertical takeoff and landing vehicle with an altitude ceiling of $15 \mathrm{~km}$, range of $\mathbf{5 0}$ $\mathrm{km}$, endurance of 3-4 months, payload capacity of $25 \mathrm{~kg}$, and a gross mass of $400 \mathrm{~kg}$ as a future Titan aerial platform.
\end{abstract}



\section{Introduction}

$\mathrm{T}$ HIS paper presents a nuclear propulsion concept designed to enable a long duration mission by an exploratory space probe to Saturn's moon Titan. The first half of this discussion considers the theoretical background of the propulsion concept and outlines the expected available power. The remainder of the discussion covers the development and testing of a physical "proof of concept" prototype in the spring of 2005. Finally, conclusions are drawn from the test results and the potential use of this power system to planetary exploration of the outer solar system.

\footnotetext{
* Graduate Research Assistant, Guggenheim School of Aerospace Engineering, Georgia Institute of Technology.

${ }^{\dagger}$ Professor, Guggenheim School of Aerospace Engineering, Georgia Institute of Technology.

* Graduate Research Assistant, Guggenheim School of Aerospace Engineering, Georgia Institute of Technology.
} 


\section{Powered Flight on Titan}

Saturn's moon Titan may hold the secret to the origin of life on Earth. Titan, the Solar System's second largest moon, has an atmosphere that is full of nitrogen and without oxygen; much like Earth's primordial state. Therefore, exploration of Titan could answer questions of how life may have originated on Earth. Titan also has a very dense atmosphere with an orange haze, obscuring the surface from the view of orbiting satellites. Therefore, to explore Titan and unlock the secrets of this mysterious planet, a form of aerial vehicle will be needed. A vehicle with vertical takeoff and landing (VTOL) capabilities would provide excellent mobility on the planet, giving a science platform the ability to explore craters, and mountains.

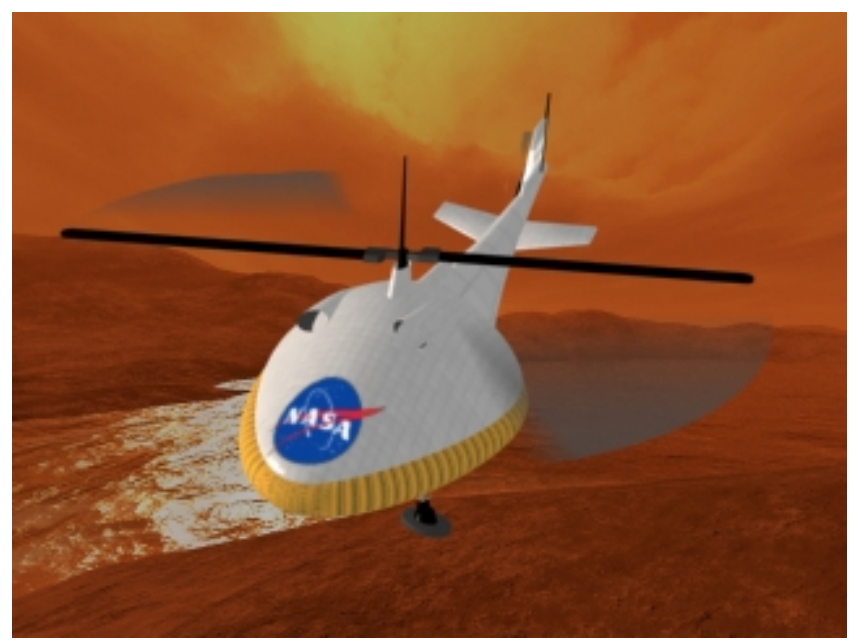

Figure 1. Titan Helicopter concept proposed by SSDL Lab at Georgia Tech

The Space Systems Design Laboratory (SSDL) at the Georgia Institute of Technology was tasked with designing a helicopter capable of the aforementioned mission goals. Please see paper by R. Prakash et al, for details on this vehicle. ${ }^{\S}$ The helicopter was designed to have an operational endurance of between 1 and 3 months and perform a cruise mission for 50 $\mathrm{km}$ at an altitude of $10 \mathrm{~km}$ several times. It was quickly realized that there would be a large power requirement to achieve these ambitious mission goals. Therefore it was imperative that a new propulsion concept be developed to enable this mission to succeed, as the conventional propulsion means of solar, chemical or battery power were determined to be inadequate.

Due to Titan's distance from the sun and dense atmosphere, solar power options were impractical. Chemical propulsion could not achieve the required mission duration and repeated flights within the mass and volume budget for the mission. The final conventional option considered was the use of lithium batteries trickle charged from a single MMRTG nuclear power source. However, after evaluating this propulsion option, it was determined that the mission down time required to recharge the batteries would be on the order of 60 days, which was deemed unacceptable. Thus a higher energy density power system had to be devised. The solution arrived at was to simply utilize the in-situ atmosphere of Titan in a "gas expander cycle" turbo machinery system. The gas expander cycle, akin to the expander cycle rocket engine, heats up the $-180^{\circ} \mathrm{C}$ Titan atmosphere $(99 \%$ Nitrogen) to $195^{\circ} \mathrm{C}$ using waste heat from two NASA-developed multi-mission radioisotope thermoelectric generators (MMRTG's). The expanded Nitrogen then runs through a turbine which is linked to an alternator to produce the necessary power.

\section{Benefits of Nuclear Gas Turbine Propulsion System on Titan}

The benefits of a nuclear gas turbine propulsion system on Titan are numerous. No other power system option considered was able to perform a long duration mission requiring months of operation with many hours of flying time during the mission, without significant down time between flights. In contrast, with the proposed system, mission duration is no longer a function of battery life or stored propellant reserves. In theory, such a nuclear gas turbine powered helicopter could fly without landing for several months unencumbered by energy consumption problems.

\section{Theoretical Background of System}

The nuclear gas turbine propulsion concept utilizes one of NASA's proposed Multi-Mission Radioisotopic Thermoelectric Generators (MMRTG) as a heat source (Figure 2).

\footnotetext{
$\S$ Prakash, R., et. al., "Design of a long endurance Titan VTOL System," AIAA Space 2005 Conference, (to be published.)
} 


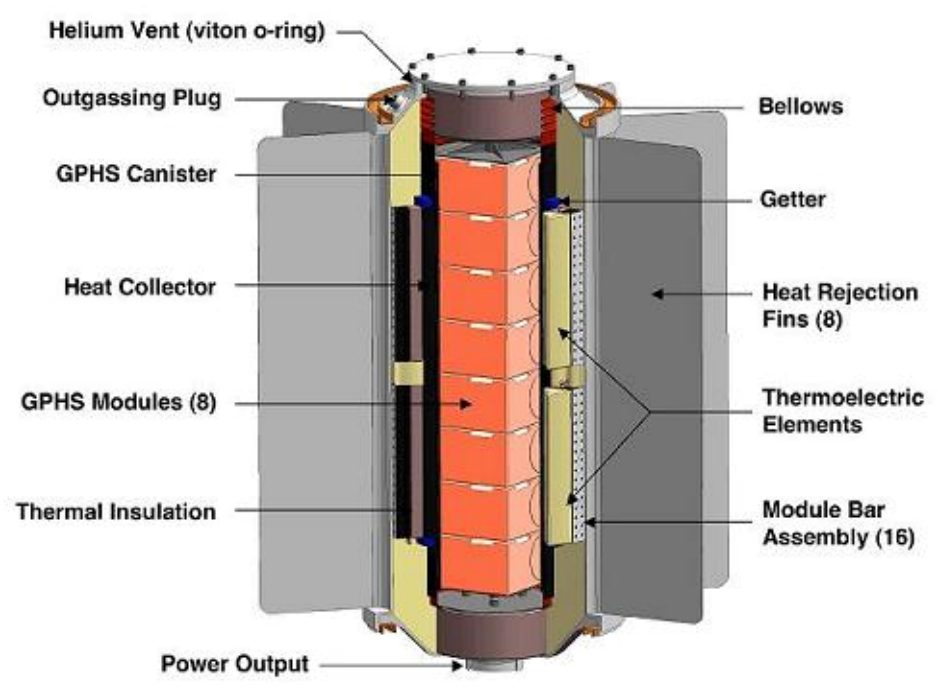

Figure 2. NASA MMRTG Schematic.
The MMRTG as proposed will consist of 8 General Purpose Heat Sources (GPHS) containing radioactive Plutonium 238 (Pu-238.) Each GPHS contains approximately $500 \mathrm{~g}$ of $\mathrm{Pu}-238$ fuel. One gram of fuel radiates approximately 0.55 Watts (W) of thermal power. Thus as proposed the MMRTG is anticipated to have a total thermal output of $2250 \mathrm{~W}$. The MMRTG utilizes this heat via the thermoelectric effect to extract electricity from thermocouplelike material. Regrettably, while this system is very reliable it is only $6-7 \%$ efficient, producing approximately 110 $\mathrm{W}$ of electrical power. The remaining $2140 \mathrm{~W}$ of power is radiated away as heat via large radiator fins.

In the proposed concept these MMRTG radiator fins would be removed and replaced with a heat exchanger loop that would capture this waste heat and use it to heat gas from Titan's atmosphere to $195^{\circ} \mathrm{C}$. (The casing surface temperature at the base of these fins is a constant $200-210^{\circ} \mathrm{C}$.) The added heat then enables power to be extracted from the gas by a turbine. The gas expander system proposed is essentially a typical gas turbine system utilizing a Brayton like cycle where the gas is compressed, heat is added, and finally the

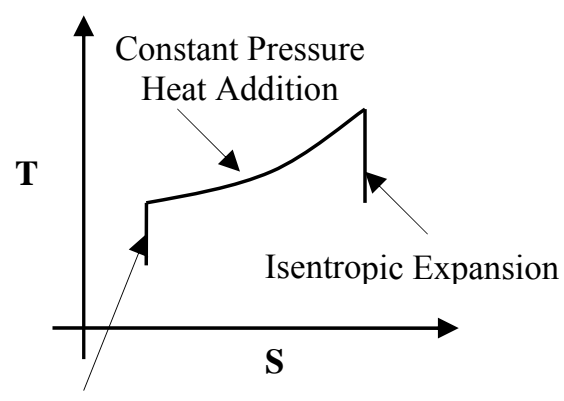

Isentropic Compression

IDEAL SYSTEM

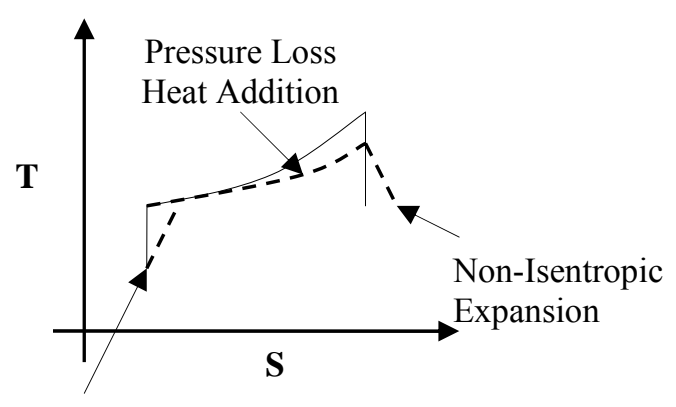

Non-Isentropic Compression

Figure 3. T-S Diagram for Gas Expander system proposed gas is exhausted through a turbine. See Figure 3 for T-S diagram.

The gas turbine type selected for this concept is a centrifugal turbine and compressor, similar to a turbocharger. A centrifugal configuration is ideally suited to this concept because the low power levels (less than 5 kilowatts) and correspondingly low mass flow rates are too small for an axial flow gas turbine. Additionally, the centrifugal configuration is easily connected to a large heat exchanger that does not have to be mounted axially between the compressor and turbine as in an axial gas turbine. Finally, as this system is being used to generate electrical power, it is quite convenient to simply fill the space between the compressor and turbine with a bearing section that doubles as a brushless alternator for extracting electrical power. In this way no gear linkages are required to convert the shaft work into electrical power since the bearings that support the compressor and turbine also hold the alternator rotor and the brushless stator is simply part of the bearing casing. See Figure 4 for a notional drawing of the conceptual system. 


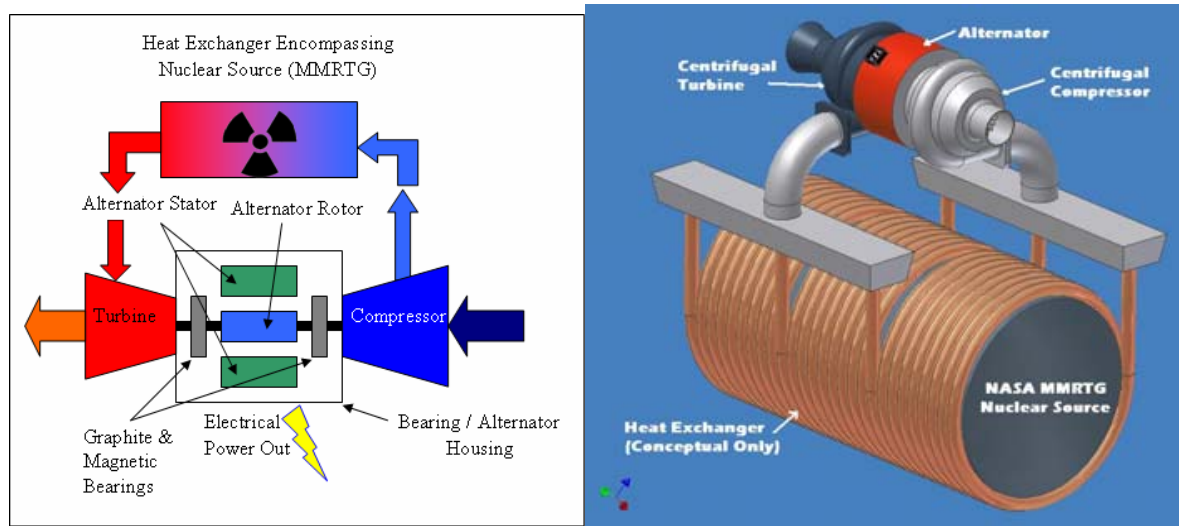

Figure 4. Conceptual drawing of Gas Expander power system

In analyzing the performance of this concept, each individual component was considered and the flow characteristics determined at the inlet and exit. The compressor and turbine were analyzed utilizing gas turbine cycle equations. Compressor sizing is relatively simple and involves determining

the required power to compress the gas. The shaft power required for a centrifugal compressor may be found from the following equation:

$$
P_{\text {shaft }}=\dot{m} U\left(c_{\theta 2}-c_{\theta 1}\right)
$$

where $\mathrm{U}$ is the rotor tip speed and $\mathrm{c}_{\theta}$ is the tangential component of the absolute velocity of the gas at the inlet (1) and the outlet (2).

Also, the ratio of stagnation pressures through the centrifugal compressor stage may be found by:

$$
\frac{P_{03}}{P_{01}}=\left[1+\frac{\eta_{\text {stage }} U\left(c_{\theta 2}-c_{\theta 1}\right)}{C_{P} T_{01}}\right]^{\frac{\gamma}{\gamma-1}}
$$

where $\mathrm{Cp}$ is the specific heat of the gas, $\mathrm{T}_{01}$ is the inlet stagnation temperature, and $\eta$ is the stage efficiency of the centrifugal compressor. Combining these two equations one can determine the power required for a given pressure ratio as:

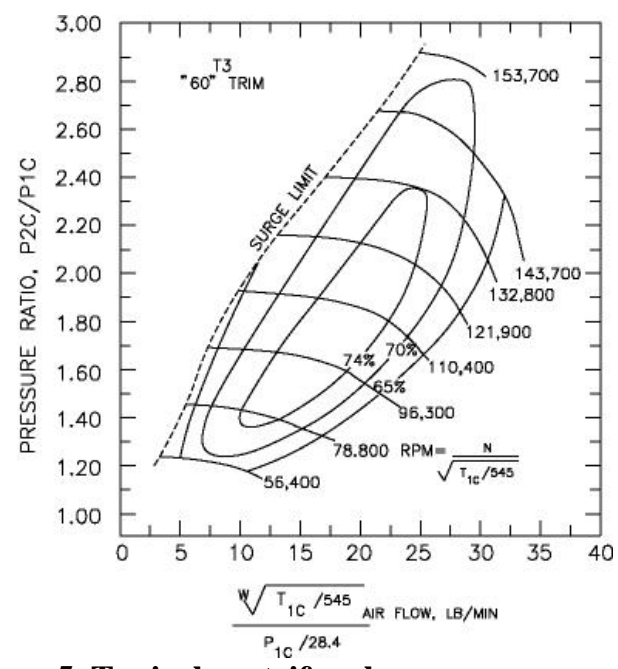

Figure 5. Typical centrifugal compressor map

$$
P_{\text {shaft }}=\frac{1}{\eta_{\text {stage }}} \dot{m} C_{P} T_{01}\left[{\frac{P_{03}}{P_{01}}}^{\frac{\gamma-1}{\gamma}}-1\right]
$$

With this equation and a corresponding mass flow, one can find the available pressure ratio and stage efficiency off a compressor map for an "off the shelf" compressor that operates in the power range desired. See Figure 5 for a representative compressor map of a centrifugal compressor in the required range.

The analysis of the heat exchanger involved using a finite discritization method to compute the change in gas properties at incremental steps along the length of the heat exchange tubing. The details of this analysis may be found in the appendix. This analysis provided the expected temperature, pressure and velocity changes in the heat exchanger tubing. It should be noted that the expected pressure drop across the heat exchanger length of tubing was computed to be on the

order of 2.0-3.0 pounds per square inch (psi).

The turbine power available can be computed from the following equation: 


$$
P_{\text {Avail }}=\eta_{T} \dot{m}_{T} C_{p} T_{\text {inlet }}\left[1-\left(\frac{1}{p_{\text {T-ratio }}}\right)^{\frac{\gamma-1}{\gamma}}\right]
$$

where in the above equation, $\dot{m}_{T}$ is the mass flow into the turbine, $C p$ is the gas specific heat, $T_{\text {inlet }}$ is the gas inlet temperature, $\eta_{T}$ is the turbine efficiency and $P_{T}$ is the pressure ratio across the turbine. Thus the power required by the compressor, the effect of heat addition in the heat exchanger and the turbine power available are all known. Iterating on the design variables, the system power required and excess power available may be computed. This theoretical sizing analysis predicted approximately $530 \mathrm{~W}$ of power available. This represents a system efficiency (assuming $2200 \mathrm{~W}$ input power,) of $24.1 \%$. In fact, if one simply considers the theoretical efficiencies of each stage in the above system (assume compressor is $72 \%$ efficient, heat exchanger is $95 \%$, turbine $66 \%$, and alternator $85 \%$ ) the overall system efficiency is theoretically $38 \%$. Thus the system performance should yield a $24 \%$ to $38 \%$ efficiency. It should be noted that all the assumed efficiencies above are within the limit of technology today if properly engineered for this specific purpose.

One other important point to note is that this system only works thermodynamically because the atmosphere on Titan is so cold and dense that it is just above the boiling point of liquid nitrogen, namely $-185^{\circ} \mathrm{C}, 5.8 \mathrm{~kg} / \mathrm{m}^{3}$ mean density and $22 \mathrm{psi}$ "sea level" pressure. If the atmosphere on Titan were to be warmed even $50-60^{\circ} \mathrm{C}$, the resultant reduction in the density of the gas would render this system impractical. Thus, this system is uniquely suited to use in space exploration on gas giant moons and planets where the atmosphere is very cold and dense.

According to this theoretical analysis, the power available from this propulsive concept, is a five fold increase over that available from a conventional MMRTG. In addition, this concept has dual purpose utility in that the MMRTG can still function effectively during the trip out to Titan or while on the ground in its conventional configuration, providing the spacecraft with $110 \mathrm{~W}$ of electrical power. Then when powered flight is desired, instead of dispersing the waste heat with heat piping, the heat is simply tapped to provide power to the gas turbine system as discussed above.

\section{Experimental Proof of Concept: Objectives \& Apparatus}

\section{A. Turbo-machinery (Turbocharger) \& Alternator}

The experimental proof of concept developed was designed to be a simple low cost method of acquiring experimental data on a new propulsion concept that would otherwise have remained merely a design concept. With this in mind, the proof of concept was made as a cost effective analog. A low cost gas turbine had to be found that would be low cost but still be able to perform the desired mission. Fortunately, lots of research in recent years has been put into improving automotive turbochargers for performance race cars, and as it turns out the HoneywellGarrett Corporation makes an excellent series of centrifugal turbochargers with relatively high efficiency. As a result a Garrett GT25R ball bearing turbocharger was selected as the gas turbine in the proof of concept prototype. See Figure 6.

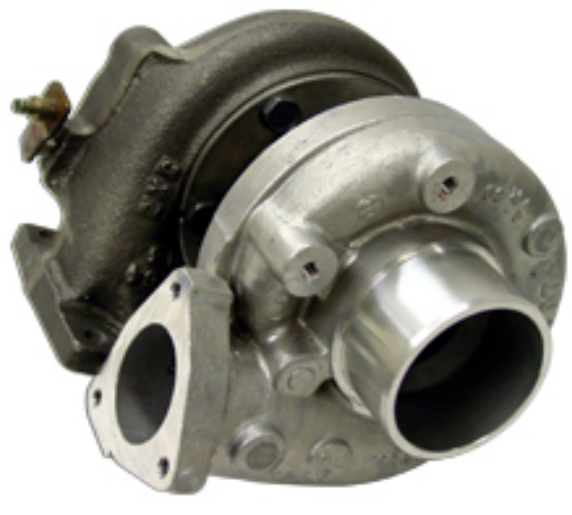

Figure 6. Garrett GT25R Turbocharger

The GT25R is the smallest of a new line of ball bearing turbochargers that incorporate high speed ceramic ball bearings instead of the conventional oil floating brass bushings used in most turbochargers. The GT25R is sized for flow rates a bit larger than envisioned for the Titan gas expander, but this tradeoff was deemed acceptable given that the turbocharger had to be of ball bearing design. The reason for this is that in order to demonstrate useful work could be extracted from the system an alternator had to be connected to the turbine shaft. In the theoretical concept, the alternator would be of a brushless type incorporated directly into the bearing housing. However, since this would require custom machining and balancing to achieve, with an "off the shelf" GT25R, an alternate solution that utilized a high speed brushless DC racing motor, was devised. (A brushless motor was used since this device can become an alternator/generator by simply spinning the shaft instead of providing current into the motor as is done in normal operation.) A high speed 
motor was required because the centrifugal turbocharger spins at extremely high RPMs. In fact, the GT25R is capable of speeds exceeding 110,000 RPM. Fortunately, for the flow rates being considered in the experiment the rotation rate is approximately $60,000 \mathrm{RPM}$, which is within the performance limits of brushless motors. The motor ultimately selected was the Hacker B50 7L which is rated for $600 \mathrm{~W}$ of continuous power at 17 Volts and 35 Amps and a nominal rotation rate of 60,000 RPM. This motor would be aligned with the inlet of the turbocharger compressor and connected directly to the turbine shaft by means of a custom linking shaft. See Figure 7 for drawing and developed hardware.

The linking shaft was threaded to take the place of the left handed nut that normally holds the compressor "wheel" onto the shaft. Alignment for this concept is critical as the turbocharger and motor spin at upwards of 60,000 RPM. This alignment was achieved with a custom inlet housing that holds the DC motor in place while allowing the inlet gas to pass around it and into the compressor. It is this customization that required a ball bearing turbocharger, as the free floating bushing design would not permit accurate alignment of the two shafts and would ultimately result in bearing failure at high rotation rates.

As a result, the flow into the compressor inlet is now forced to flow around an obstruction instead of smoothly flowing down a straight tube. Thus, before hardware could be built, a simulation of the customized turbocharger inlet was performed to ensure the flow obstruction would not be a problem. Utilizing simple incompressible Navier-



Figure 7. CFD Solution of velocity field superimposed on custom inlet geometry \& Completed system with motor connected to turbine shaft
Stokes modeling in the Computational Fluid Dynamics (CFD) program Femlab 3.0, the flow velocity and pressure field were computed. Figure 7 is an overlap of the CFD velocity profile solution on the proposed custom turbocharger inlet geometry and the final hardware.

The analysis showed that the flow velocity would increase by approximately $15-20 \%$ over the inlet velocity as a result of the flow restriction and would produce only a 0.5-1 psi pressure drop. This was deemed acceptable, enabling further development.

In addition, because the alternator location exposed it to cryogenic temperatures, the motor was wrapped with a strip heater coil connected to a power controller keeping the motor casing warm enough to prevent failure.

\section{B. Simulated Nuclear Source and Heat Exchanger}

With the gas turbine and alternator selected, the remaining major component to be created was the simulated MMRTG or SMMRTG and the heat exchanger. The SMMRTG was welded together from thin carbon steel for simplicity and made to have the same external dimensions as the MMRTG. (The MMRTG casing dimensions are approximately 14.5 inches in diameter (excluding the radiator fins,) and 26 inches in length.) Then six 500 Watt, $120 \mathrm{~V}$, power controlled, $\mathrm{U}$ bend heater coils were inserted to generate the necessary heat to simulate the MMRTG surface temperature of $200^{\circ} \mathrm{C}$. Finally, as the space required by 6 heater coils is significantly less than the actual internal components of the real MMRTG, the heater coils were surrounded by an annulus of $70 \mathrm{lbs}$ of magnesium oxide powder to simulate the heat capacity or "thermal mass" of an MMRTG made of aluminum, lithium, plutonium, thermoelectric material and insulation. Finally, the remaining empty interior volume was filled with fibrous insulation to prevent heat leakage into the air. See Figure 8 for a photograph of the SMMRTG. 


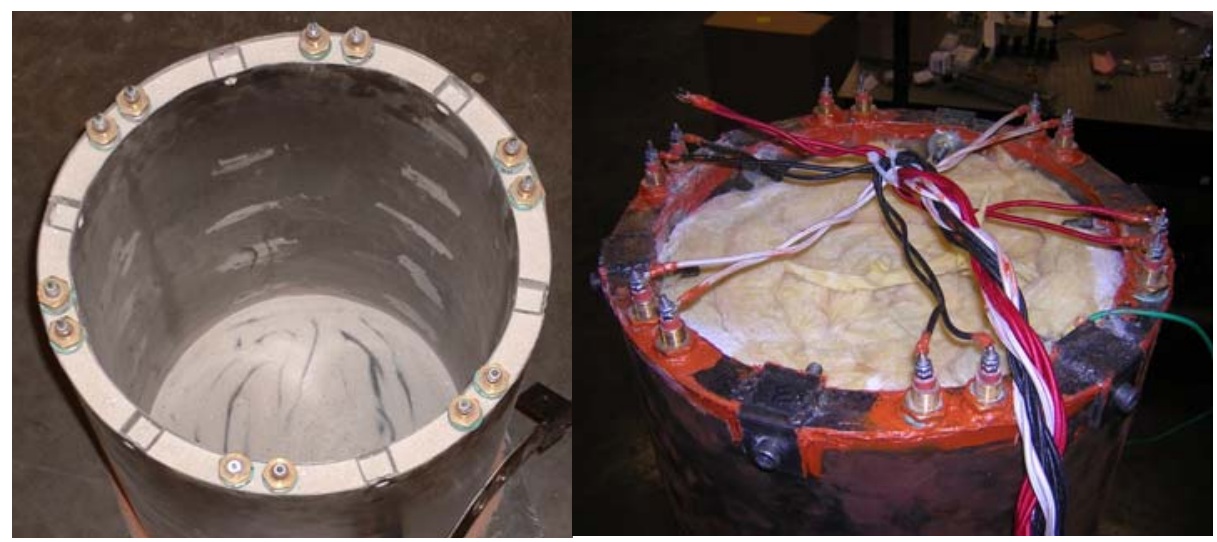

Figure 8. SMMRTG Casing with heater coils, MgO sand, and fibrous insulation

The next part of the proof of concept to be created was the heat exchanger tubing. The theoretical analysis performed indicated that three 1" pipes would be sufficiently long, if wrapped around the SMMRTG exterior, to be able to each heat the gas to the desired exit temperature. By diverting the flow into three separate tubes, the heat transfer could be improved beyond what a single larger diameter tube could achieve. Additionally, the triple tube configuration saved construction cost and simplified flow control with simple solenoid valves in a $1 / 3^{\text {rd }}, 2 / 3^{\text {rd }}, 3 / 3^{\text {rd }}$, flow rate fashion. More complex and efficient heat exchanger geometries were considered, but due to time and cost constraints were discarded for the proof of concept system. Figure 9 shows the SMMTRG with the heat exchanger coils being installed. Regrettably, Figure 9 also shows the coil wrapping problems encountered with the system to be discussed later).


Figure 9. SMMRTG with heat exchanger coils \& SMMRTG with solenoid valves and thermal insulation

\section{Data Acquisition Equipment}

The next major component of the proof of concept system to be discussed is the data acquisition and sensor array setup. As this entire proof of concept is for the purpose of validating the system concept it was important that good temperature and pressure data be obtained at the inlet and exit of each system component. In order to accomplish these data acquisition requirements four thermocouple probes and three pressure transducers were used.

The pressure transducers used are $0-30 \mathrm{psi}$ gauge (psig), 1-5 Volt output transducers which provide strong and clearly readable low noise signals without the need for extra signal conditioning and amplification. The thermocouples used are of the type $\mathrm{K}$ variety as these thermocouples can read temperatures as cold as $-200^{\circ} \mathrm{C}$ and as hot as $1800^{\circ} \mathrm{C}$ which fully spans the temperature range of this system. The thermocouple signals are conditioned with four signal conditioners, two specifically designed for cryogenic temperature ranges from -200 to $0^{\circ} \mathrm{C}$ and two for normal temperature ranges from 0 to $300^{\circ} \mathrm{C}$. The sensors are located at four key points in the system, namely, the compressor inlet, compressor exit, and turbine inlet and turbine exit. The compressor exit and turbine inlet also double as heat exchanger inlet and exits respectively. At the compressor inlet, exit and turbine inlet both the stagnation temperature and stagnation pressure are read. While at the turbine exit only the temperature is recorded since the turbine exhausts to Earth atmospheric pressure. The locations of the sensors are indicated on the finished hardware in Figure 10. 




Figure 10. Sensor Locations on hardware



Figure 11. Data signal conditioning and acquisition box boards.
The data acquisition hardware utilized for reading in the signals from the pressure transducers and thermocouple signal conditioners are two National Instrument USB-6009 12-Bit USB DAQ boards. These boards are capable of sampling rates up to 10,000 samples per second in 12-Bit or 4096 discrete increments. Given the span of 1-5 Volts for a 0-30 psi range on the pressure transducers, this translates into a minimum pressure resolution of 0.038 psig. Since the minimum resolution of the Omega PX-181 pressure transducers used is $+/-0.3 \%$ Full Scale or $0.09 \mathrm{psig}$, the DAQ board resolution is not an issue. In any case a pressure resolution on the order of $0.1 \mathrm{psig}$ is sufficiently accurate for these tests. For the thermocouple signal conditioners the output is $0-10$ Volts and thus the minimum resolution the 12-Bit boards can resolve is $0.307^{\circ} \mathrm{C}$, but again the minimum accuracy of the signal conditioners is $+/-1{ }^{\circ} \mathrm{C}$, more than sufficient for the experiments being conducted. Figure 11 is a photograph of the data acquisition box created for the experiment to house and protect all the power supplies, signal conditioners and DAQ

The data read in from these 7 sensors was imported into a Labview code written to enable the user to see the temperature and pressure readouts in real-time during a test on easy to interpret graphs and dial gauges. Additionally, while the data are being displayed to the screen, they are saved to a buffer and exported to an Excel spreadsheet at the end of each test run. The sampling rate used in all tests was 10 samples per second which provides sufficient temporal resolution to observe transient effects without creating overly large data sets.

\section{Turbocharger Specific Support Systems}

One element of the proof of concept system that had to be implemented due to use of off the shelf hardware was the cooling and lubrication system installed for the turbocharger. Despite being a ball bearing configuration turbocharger, the GT25R still requires a low flow rate oil supply to ensure the ceramic bearings remain lubricated. Thus, an oil pump and filter were incorporated into the proof of concept to ensure the turbocharger bearings received the proper lubrication. The secondary auxiliary system installed for the turbocharger was a water cooling pump and liquid reservoir. In normal turbocharger operations there is a cooling jacket around the bearing assembly which helps to keep the bearing casing cool, but in this application the cooling jacket was actually used to keep the bearing assembly warm. That is to say that the compressor side of the system is exposed to $-180^{\circ} \mathrm{C}$ temperatures and so the water cooling jacket helped to maintain the required thermal gradient between the hot turbine side and the cold compressor side. See Figure 12 for a picture of the two pumps and anti-freeze liquid reservoir utilized in the test setup. 




Figure 12. Oil and anti-freeze cooling lines for turbocharger bearing assembly

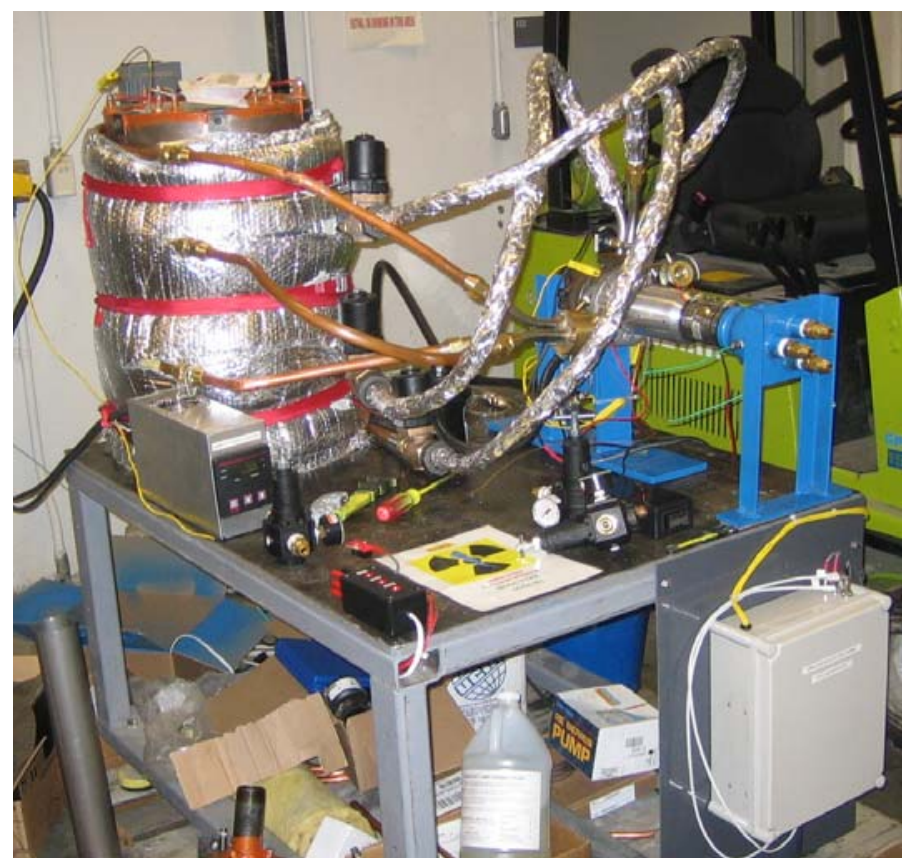

Figure 13. Complete Gas Expander Proof of Concept system

\section{E. Simulated Titan Atmosphere}

Simulating Titan's very cold, dense, 99\% nitrogen atmosphere was accomplished by utilizing 180 Liter, 230 psi liquid nitrogen Dewars. Gas flow pressure regulators were used on the tank gas outlets to provide low pressure cryogenic nitrogen gas at approximately -180 to $-190^{\circ} \mathrm{C}$. The 230 psi versions of the liquid nitrogen Dewars were utilized because the 22 psi versions were not capable of supplying a continuous gas flow without massive pressure oscillations.

\section{F. Complete System}

An assembled picture of the integrated system is presented in Figure 13. This system represents a development program in which a system concept was taken from theoretical idea to functioning hardware in less than three months and for approximately $\$ 9,000.00$.

\section{Test Results}

The first tests conducted centered on determining the answer to some basic feasibility and safety questions. These tests were labeled 1000 series tests and involved some of the system components discussed above and some substitute components. The second test series were labeled the 2000 series tests and involved testing the complete system as seen in Figure 13 above.

\section{G. 1000 Series Test Results}

In the 1000 series tests, basic questions were answered utilizing the completed SMMRTG and heat exchanger and an old "scrap" turbocharger. This test series was primarily focused on answering the question: Can an automotive turbocharger not rated for cryogenic temperatures, survive the thermal environment being imposed upon it? The answer to this question was an important one to know before a brand new $\$ 1,000$ GT25R was connected to cryogenic gas. The concern was that the compressor stage may be made out of inferior grade cast aluminum and might develop cracks from the thermo cycling. Furthermore, the rotating seals were not designed for the thermal operating regime and it was possible that they might leak or seize up. An old Schweitzer turbocharger that had reached the end of its rated design life, but was still functional, was connected to the heat exchanger. Then gas from a single 180 Liter (L) Dewar was supplied to the turbocharger inlet. Apart from the purely empirical results of turbocharger survival, the test series was also designed to get a first look at the performance of the heat exchanger and test the DAQ system. With the aforementioned goal in mind, one thermocouple and pressure transducer were connected to the system at the compressor exit and another one of each at the turbine inlet. The results of these tests are summarized by the data taken from test 1002, which is given in Figure 14. 

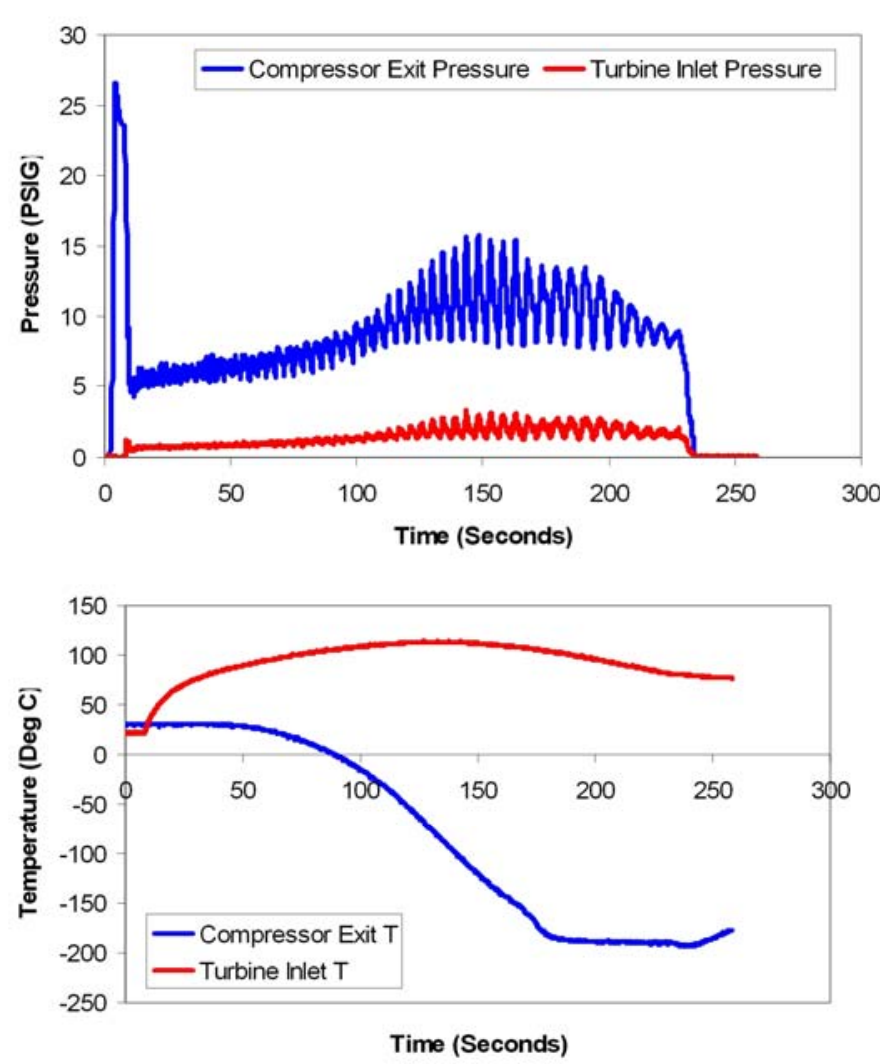

Figure 14. Pressure and Temperature data vs. time for test 1002

Note that the inlet pressure is highly unstable and suffers from pressure oscillations. These pressure oscillations are caused by the fact that the single $180 \mathrm{~L} \mathrm{LN}_{2}$ Dewar was not capable of supplying the flow rate of gas desired at a constant pressure. This effect is a limitation of the Dewar that is inherent to all cryogenic tanks, and results from the fact that as the gas phase is being tapped off, the gas pressure drops until the point at which liquid phase nitrogen boils off to return the pressure to equilibrium. The higher the flow rate of gas leaving the system the larger the pressure oscillations will be. Thus the key lesson learned from this test was that more than one Dewar will be needed for the final test configuration. (Ultimately three Dewars were used so that each one would only have to supply $1 / 3^{\text {rd }}$ the gas and thus have a correspondingly smaller pressure oscillation.)

The other major result obtained from the pressure data was that the pressure drop across the heat exchanger loop was much greater than originally anticipated. The average pressure loss for this test was on the order of 10 psi and the theoretical pressure loss envisioned was only 2-3 psi. The pressure loss issue is important because if the pressure loss is too great the compressor will not be able to raise the gas pressure sufficiently to offset the losses and the turbine will not be able to extract work. Fortunately, the primary cause of this excessive pressure drop was determined to be the piping fittings used in this test. These fittings or "merge collectors" were created to take the 1.5" compressor exit pipe and merge it into the three $3 / 4$ inch pipes of the heat exchanger and the reverse on the turbine inlet side.

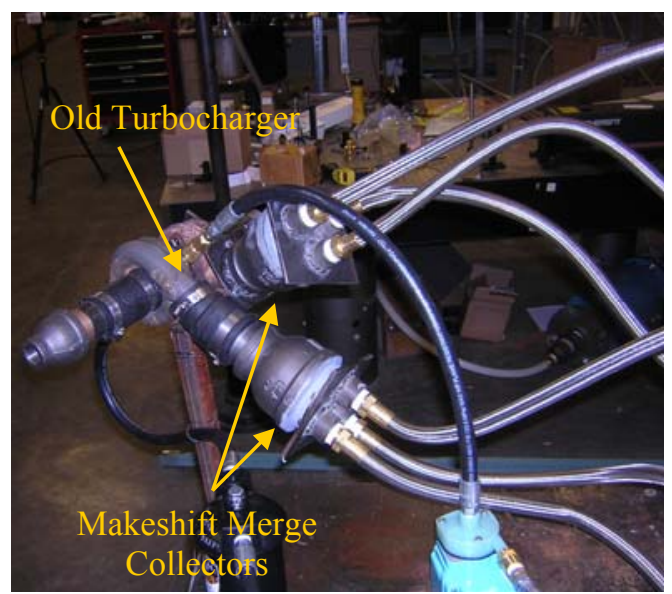

Unfortunately, these merge collectors were crudely improvised from pipe junctions (see Figure 15).

These merge collectors had to be replaced and custom motor mount machining and improved merge collectors were machined. The other major result obtained from the 1000 series of tests was that the temperature data showed a low efficiency for the heat exchanger. The design output temperature for the heat exchanger was $195-200^{\circ} \mathrm{C}$ and as may be clearly seen from Figure 14 the temperature did not exceed $110^{\circ} \mathrm{C}$. The reason for this problem was the construction of the heat exchanger coils. Regrettably, the copper tubing utilized in the heat exchanger loops was a soft copper that ships in large coils, which are cumbersome to work with. Thus, for this first heat exchanger loop, the tubing was first straightened out before bending around the SMMRTG casing. The undesired result of this process was that the copper was workhardened and could not be made to wrap easily around the casing. As a result the copper tubing for the bottom coil did not completely touch the casing all along its entire length, which resulted in significantly reduced heat transfer. In an effort to avoid workFigure 15. Old turbocharger and makeshift merge collectors utilized in $\mathbf{1 0 0 0}$ series tests hardening the middle coil, as happened to the first coil, the middle coil was unwrapped directly from its packaging
around the casing, thus reducing work hardening of the copper tubing. Fortunately this improved wrapping technique provided excellent coil contact with the SMMRTG casing, resulting in this coil actually operating quite well when taken alone. The top coil unfortunately was a composite coil made of half segments of the first and 
second coil so only half of it was wrapped properly with the second half having several non-contacting segments. The combined effect of this manufacturing defect was that the overall heat exchanger efficiency was lower than anticipated. While it is possible to run gas through only the middle coil to obtain higher exit temperatures, the single tube could not handle the mass flow rate requirements of the entire system. The solution to this problem, would have been to replace the top and bottom heat exchanger coils, however due to budgetary and schedule constraints this option was not feasible.

The final result from the 1000 series tests showed that the turbocharger was fully capable of surviving the harsh temperature environment of cryogenic gas flow on the compressor side, and gas temperatures exceeding the boiling point of water on the turbine side. These tests confirmed our initial theoretical assumptions. In addition, one object did fail as a result of the temperature environment; the rubber transition coupling used to connect the turbocharger to the inlet pipe. The cryogenic temperatures simply hardened and embrittled the rubber to the point of cracking, which is not terribly surprising given the low grade rubber material used. This problem was solved on the final system configuration by simply encasing the rubber in a tight metal jacket, so as to ensure that if cracks did develop they could not propagate and effect the rigidity or seal integrity of the pipe connection.

\section{H. 2000 Series Test Results}

The 2000 series tests involved the final proof of concept configuration, (Figure 13), which incorporates all the components of the system as discussed in the apparatus section. This configuration also includes the parts that were modified or changed as a result of the lessons learned from the 1000 tests, such as the improved merge collectors. In this final configuration, there were a couple of other minor components not mentioned above. The first was that the brushless motor output leads were connected to a voltage rectifier circuit, as the output from a brushless motor is a three phase power output. Because recording power readings from a three phase circuit required extra sensors, it was decided to simply connect the motor to the proper rectifier circuit and measure the voltage and current through a load on the DC side of the circuit. The other additional component of importance was the starter lithium batteries and power controller used to spin the turbine up initially. These components were simply taken from an RC plane kit.

The first test conducted with the new hardware, test 2001, produced interesting results. Figure 16 shows the temperature and pressure plots for this test vs. time. 



Figure 16. Pressure and Temperature profiles vs. time for test 2001

In this test the load system was set up correctly, but the startup motor controller was not yet installed and the voltage measurement channel on the DAQ board was not yet configured. So as a result, the amperage from the test was recorded, but the motor output voltage was not.

From Figure 16, the pressure drop across the improved piping connections is still higher than desired, but is only on average 5-6 psi or roughly half the pressure drop from the first test series. Thus, the improved merge collectors were beneficial, but not to the extent hoped for. The other pressure related issue was that in order to achieve the desired flow rate, the inlet pressure was noticeably above the ambient pressure and hence to some extent the system was being pressure driven. That is to say that the work being extracted from the flow is not entirely due to the heat addition from the simulated nuclear source, and is partially due to a positive pressure differential between the system and the atmosphere. However, due to the pressure drop through the heat exchanger tubing, the pressure at the turbine inlet is only slightly above where it would have been had the system been operating correctly.

The other interesting result of this test was that the output power generated, turned out to be more than the soldered leads on the voltage rectifier circuit could handle. Before the leads melted off, at approximately 200 seconds, the amperage reading was steady at approximately 22 Amps. Given the resistance in the DC circuit was $1.2 \mathrm{Ohms}$ and that power may be found from $\mathrm{P}=$ $I^{2} R$, the mean power output was then $\mathbf{5 8 0 . 8}$ Watts. As mentioned above, some of the reason for this power output is due to the artificial pressure gradient caused by the inlet pressure rise. However, as the power measured is dependent on the turbine pressure ratio alone, this number is a reasonable representation of the amount of power that a system properly operating could produce. That is to say, if the compressor were operating at a pressure ratio of approximately 1.6 to 1.8 , as designed, and the pressure drop across the heat exchanger was only a few psi as theoretically predicted, then this power output would match almost exactly with the theoretical power output.

Since this test was partially pressure driven, another test which would prove the system actually runs on its own, with only the heat addition from the nuclear source, was attempted. In order to get the flow rate necessary for the turbocharger to be operating within its design parameters, the flow rates from the Dewars had to be so high that the inlet pressure was significantly above ambient pressure. Several test runs were conducted to try to improve the situation, but ultimately it was decided to run a test were the flow rates were very low and concentrate on proving that the system could be self-powered. Test 2006 was one of these tests and Figure 17 is an excerpt from the data set from this test showing clearly that the system can run on heat addition alone. 

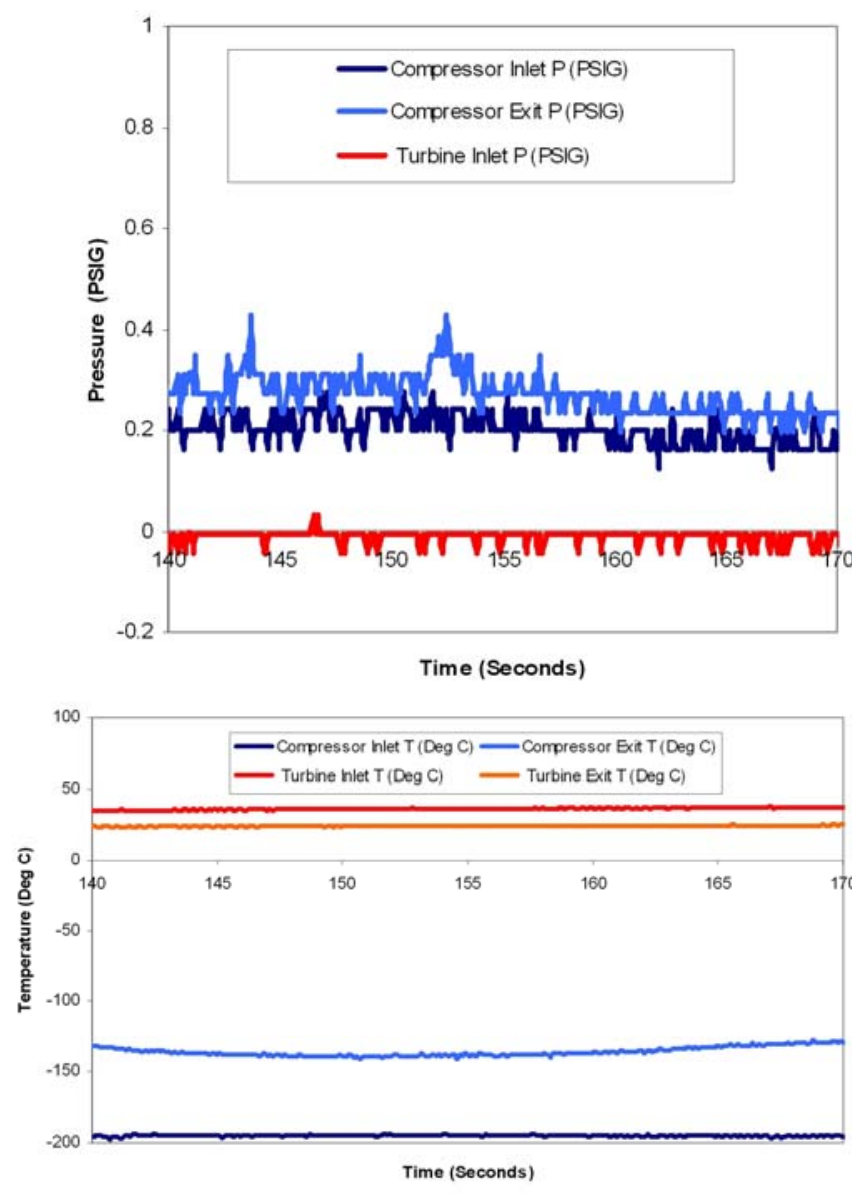

Figure 17. Pressure and Temperature data vs. time for test 2006
In test 2006, the Dewars were opened and gas allowed to flow until the gas reached the desired cryogenic operating temperatures; then the flow rate was regulated down until the turbine was not spinning. Next the new motor controller, which had just been installed, was used to spin the turbocharger up to its operating speed. Finally, the starter power was switched off and the turbine left alone. Despite the low flow rates the system was able to continue spinning on its own, demonstrating conclusively that the system concept can work. In addition to the purely empirical evidence of seeing the turbine spin, the data in Figure 17 clearly shows a small pressure rise across the compressor. While this data is near the resolution limits of the sensors as is evidenced by the discreet jumps in the data, one can clearly see a compressor exit pressure that is higher than the inlet pressure. Additionally, the temperature data shows the correct temperature drop across the turbine indicating the turbine is extracting useful work.

Thus, this test showed that the heat addition from the nuclear source will spin a centrifugal turbine.

\section{Implications for Planetary Aerial Vehicles}

The implications of this theoretical study and accompanying physical proof of concept testing for powered exploration aerial vehicles is quite significant. As the theoretical model demonstrated and the physical tests partially confirmed, it is possible to take the cold dense gas from Titan's atmosphere and utilize it to recover useful work from MMRTG waste heat that would otherwise be radiated away. The implications of this concept for Titan aerial vehicles is significant, because it means that missions of unspecified durations would be possible, thus enabling exploration of the entire planet surface over the course of several years. The Titan helicopter proposed by Georgia Tech's SSDL lab was designed to use three of these power systems in parallel to provide the necessary power required by the helicopter to fly. However, while this concept was proposed to solve the power requirement issues of a Titan helicopter it is no great stretch of the imagination to realize that this system could also be a viable power source for a Titan land based rover, an airplane or even a blimp. Indeed, one may go farther and observe that while this system was designed to work on Titan, it could easily be utilized on any of the outer gas giant planets, where the gas is sufficiently cold and dense to allow it to operate. In fact, by performing a quick sizing for each of the outer planet's atmospheres, one may see that the baseline system is capable of producing power in each atmosphere (Figure 18). 

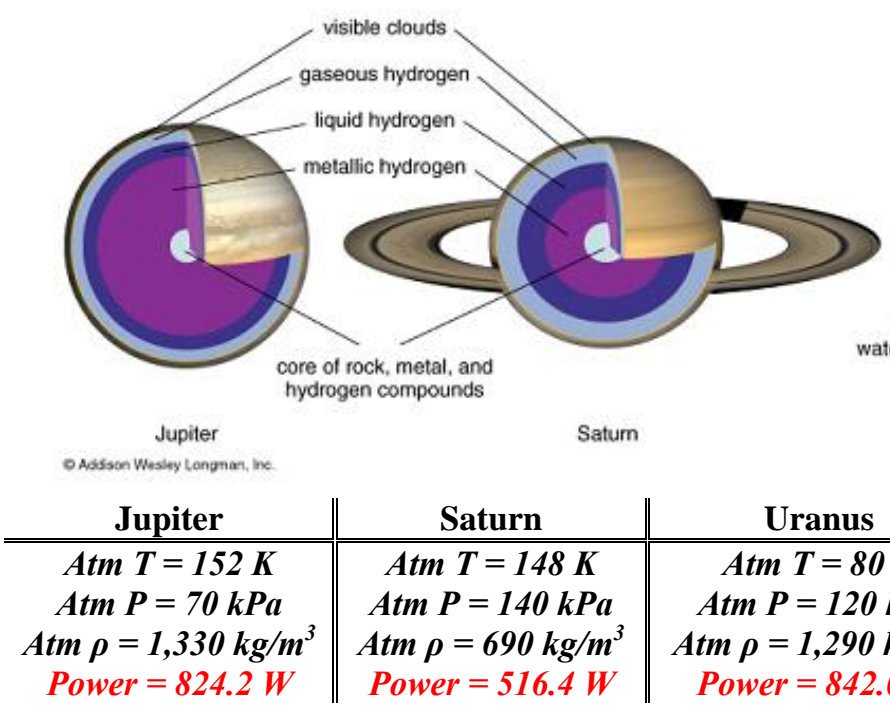

Saturn

\begin{tabular}{||c||} 
Saturn \\
\hline Atm $T=148 \mathrm{~K}$ \\
Atm $P=140 \mathrm{kPa}$ \\
Atm $\rho=690 \mathrm{~kg} / \mathrm{m}^{3}$ \\
Power $=516.4 \mathrm{~W}$
\end{tabular}

Atm

Uranus

$\operatorname{Atm} T=80 \mathrm{~K}$

Atm $P=120 \mathrm{kPa}$

Power $=842.0 \mathrm{~W}$

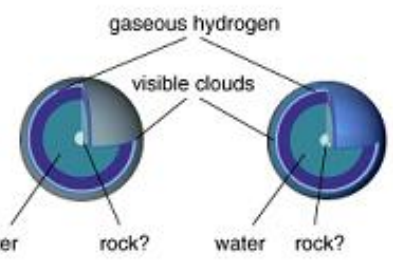

Uranus

Neptune

Figure 18. Planets of the outer Solar System \& Gas Expander Available Power
From the above data one can see that the gas giant planets have atmospheres more suited to the extraction of power (due to the higher density). So, this system, once developed for one planetary mission, could be evolved to suit many such missions involving gas powered flight through the clouds of the outer solar system gas giants.

\section{Conclusion}

In the discussion above a theoretical power system for powered flight on Saturn's moon Titan was proposed. This theoretical concept was then demonstrated by a physical proof of concept. The key results from the tests conducted were that the system was shown to be able to produce 580 Watts of measured power and while there were system inefficiencies and part of the power available was due to artificial pressure gradients, the stated result is very encouraging. In addition, subsequent tests showed that the system is capable of running purely on the heat added from the simulated nuclear source, albeit at a significantly degraded performance from the theoretical model, due to the system inefficiencies. Additional minor improvements to this system should be possible to the point of providing useful data that validates the theoretical concept very conclusively with only a modest outlay of additional time and cost.

Another useful result of this program was that it demonstrated that a purely theoretical concept can be taken from paper study to partially working proof of concept in a remarkably short period of time, (only 14 weeks) and for less than $\$ 10,000$. This rapid development and evaluation of a physical proof of concept for relatively low cost is an important aspect of engineering design.

The promise of this propulsion system concept for powered flight on the cold distant gas giants and their moons of the outer solar system are quite remarkable. This new technology is a mission enabling idea for the Titan helicopter and may provide far greater versatility for any number of aerial platforms than a conventional MMRTG alone could provide. Indeed, it is the fact that this system concept utilizes an already existing piece of hardware and simply modifies it to improve its versatility that makes this concept so interesting. It is the authors' hope that one day many robotic scout missions of the outer solar system will be possible and that perhaps one may even use the nuclear gas expander system discussed here as a power source. 


\section{Appendix}

After test 2006 a mechanical failure of the connection between the turbocharger shaft and the brushless alternator made further system testing impossible, so several subsystems tests were conducted instead. These tests were aimed at providing a greater understanding of the problems and inefficiencies encountered with the SMMRTG and accompanying heat exchanger tubing.

The first test conducted involved disconnecting the outlet side of each heat exchanger loop and measuring the exit gas temperature, given a cryogenic gas inlet temperature. For these tests a digital multimeter with thermocouple probe was used, so as to not have to dismantle the thermocouple wiring on the turbocharger. The data plots from the

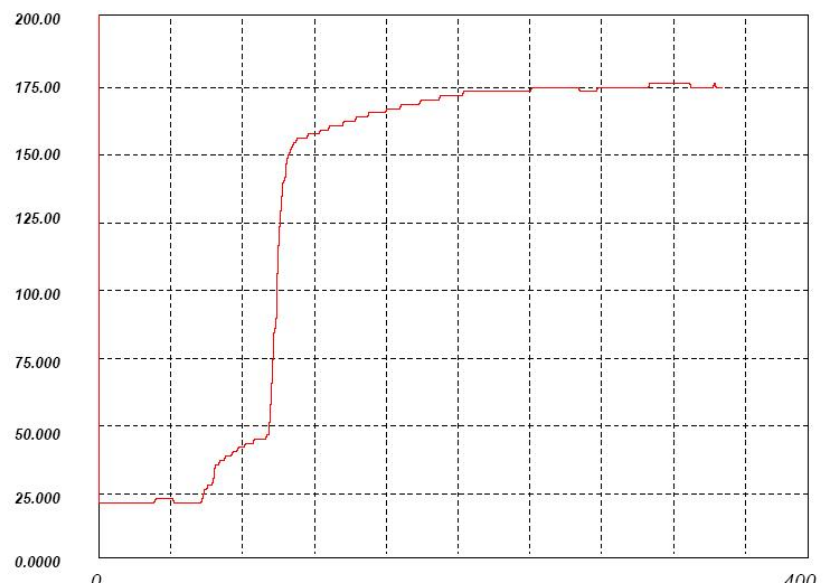

Figure 19. Temperature $\left(\operatorname{In}{ }^{\circ} \mathrm{C}\right)$ vs. Time $(400$ seconds max) for top heat exchanger coil. (Readout from multimeter)

program that interfaces with the multimeter are not terribly good so only the plot of one coil is shown in Figure 19.

As can be seen in the figure, the exit temperature of the top coil reaches $150^{\circ} \mathrm{C}$ within approximately 115 seconds and levels out at $175^{\circ} \mathrm{C}$ after about 200 seconds. Similarly although not shown, the middle coil reaches $220^{\circ} \mathrm{C}$ in a mere 50 seconds and levels out at $250^{\circ} \mathrm{C}$ after about 150 seconds. Finally, the bottom coil is only able to reach $110^{\circ} \mathrm{C}$ after a laboriously slow warm up period of some 800 seconds. Clearly, as mentioned above, the bottom coil was barely functional and in fact seriously detracted from the effectiveness of the other two, as it simply allowed cold gas to flow into the turbine.

Interestingly, from this data, it appears that the middle coil was working well within design parameters and even a bit beyond. The reason for this is undoubtedly that the temperature setting for the SMMRTG was set above the $200^{\circ} \mathrm{C}$ design point in all subsequent tests to the 1000 series tests in an attempt to offset the heat exchanger inefficiencies. What this data shows though is that if all three coils were properly wrapped like the middle coil, it might be possible to achieve turbine inlet temperatures closer to the design point of $200^{\circ} \mathrm{C}$.

The other issue that this test illustrates is that there was significant heat loss through the connecting pipes between the heat exchanger and turbine inlet, despite the thermal insulation wrapped around the tubes. If the temperatures are averaged for each coil at 100 seconds and assumed to each contribute $1 / 3^{\text {rd }}$ to the exit temperature of the heat exchanger, then one may expect the exit temperature to be on the order of $150^{\circ} \mathrm{C}$. Yet from test 1002 , which had one of the highest inlet temperatures, one may observe a maximum inlet temperature of only about $110^{\circ} \mathrm{C}$. Thus, evidently there is at least a $40^{\circ} \mathrm{C}$ temperature drop between the heat exchanger exit and the turbine inlet. Fortunately, this problem can be solved relatively simply by just shortening the connection piping between the two components, and adding additional layers of thermal insulation wrap.

The second test-run sequence, to better understand the heat exchanger system, was a test in which the pressure sensors from the compressor inlet and exit were removed and reconnected to the inlet and exit of the heat exchanger. In this test, various flow rates of gas were allowed to flow through the system, as it would during a regular test, but in this case pressure readings were taken at the immediate inlet and exit of the middle heat exchanger loop. The middle loop was selected as it was assumed this loop has the lowest pressure drop and hence should give a good estimate of the best case scenario pressure drop in the real heat exchanger. Figure 20 is a plot of the inlet and exit pressure for this test as well as the pressure drop between these two readings. 

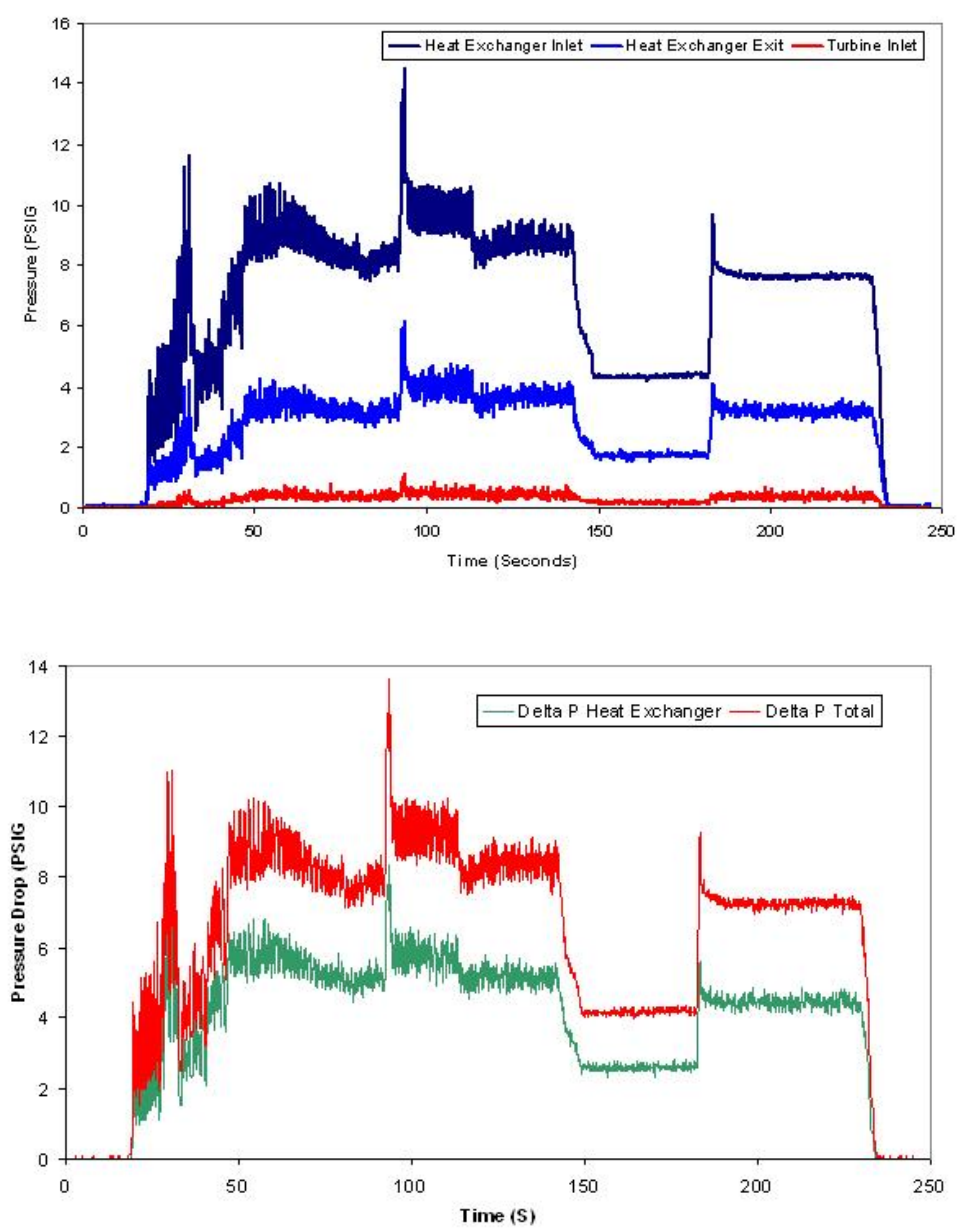

Figure 20. Pressure data for heat exchanger inlet \& exit and turbine inlet as well as pressure drop across just the heat exchanger and also the heat exchanger and connector tubing
As can be seen from the data, there was a pressure drop across the heat exchanger, as well as one between the heat exchanger exit and the turbine inlet. Remarkably, for the lower flow rate portion of the test, which corresponded to a flow rate close to the design flow rate, one may observe that the pressure drop across the heat exchanger is indeed about 3 psi. Thus, incredibly, this test demonstrated that the theoretical analysis of the heat exchanger tubing was not very far off from the actual physical model. However, the pressure drop from the exit of the heat exchanger to the inlet of the turbine is an additional 2.2 psi which was not properly accounted for in the theoretical model. Of course at the higher flow rates the pressure drop is worse, as one would expect. So this test demonstrated that here again the performance of the best heat exchanger coil on the SMMRTG was relatively close to the theoretical performance predicted. However, the additional losses caused by the extra tubing and flow merge collector connecting the heat exchanger to the turbine were not accounted for and turned out to be non-trivial. As with the temperature problem discussed above, however, this problem could be fixed relatively easily by improving the connection between the heat exchanger and the turbine inlet. Again, shorter tubes and a carefully designed and constructed third generation merge collector would improve the situation considerably. 
The simplified forms of the equations used to solve the heat exchanger problem discussed in the theoretical section are summarized in appendix table 1.

Table A1. Heat Exchanger Sizing Summary

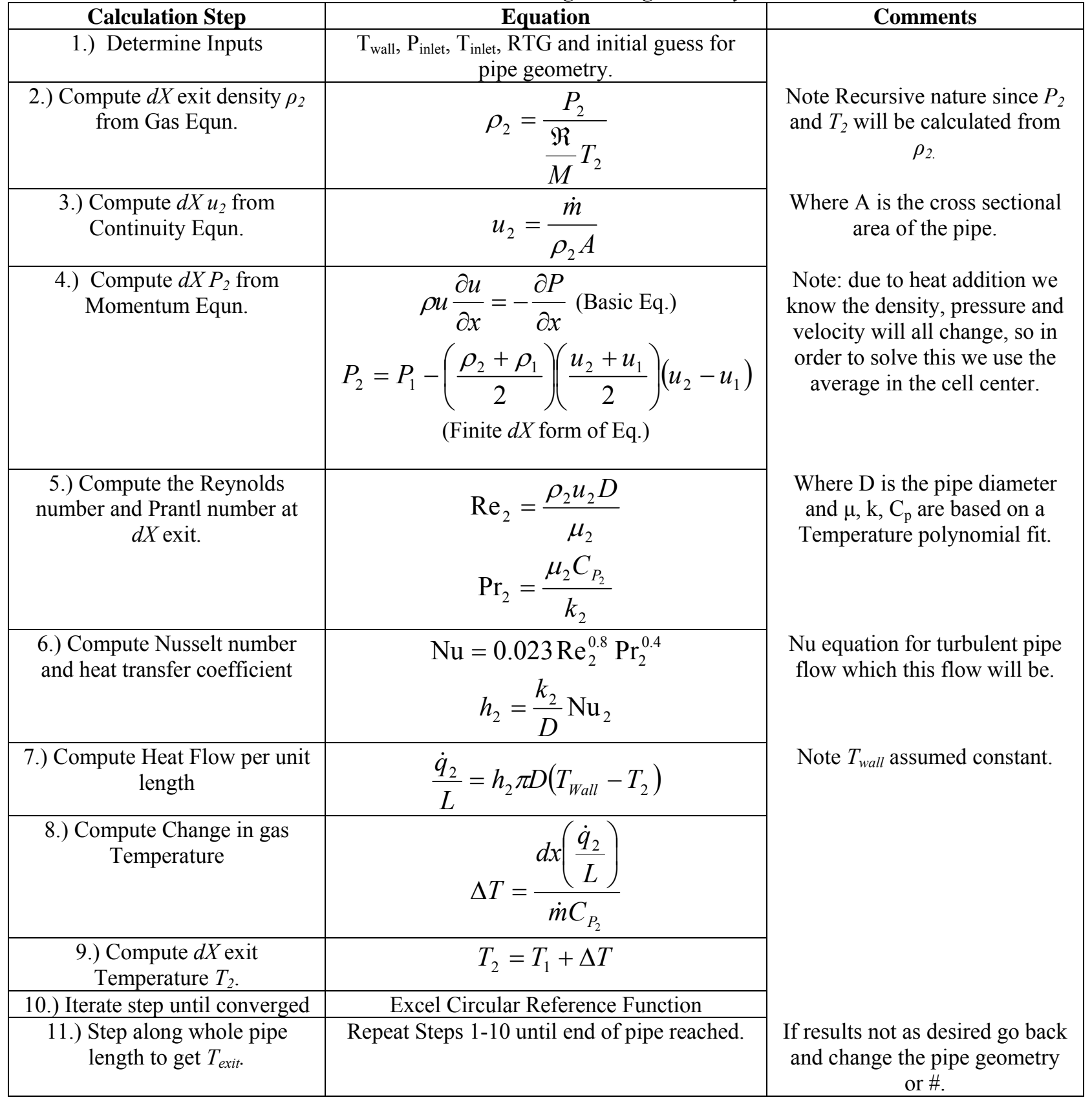




\section{Acknowledgments}

The authors would like to gratefully acknowledge all the help and support provided by the Aerospace Machine Shop machinists, Andrew Carignan, Scott Moseley, and Scott Elliot, without whose help this project would not have been possible. We would also like to thank Bobby Noble, John Holthaus, and David Scarborough of the Aerospace Combustion lab, whose help with the construction of this project was much appreciated. Finally, we would like to thank Nathan Rolander of the Mechanical Engineering Department, whose assistance in the evaluation and implementation of the initial design concept was invaluable.

\section{References}

Hale, Francis. Introduction to Space Flight, Prentice Hall, 1994.

Hill, Philip, Peterson, Carl, Mechanics \& Thermodynamics of Propulsion, Addison-Wesley Publishing, 1992.

Holman, J.P., Heat Transfer $9^{\text {th }}$ Edition, McGraw Hill, 2002.

Humble, Ronald W., Henry, Gary N., Larson, Wiley J. Space Propulsion Analysis and Design, McGraw Hill, 1995.

Moran, Michael J., Shapiro, Howard N., Fundamentals of Engineering Thermodynamics $4^{\text {th }}$ Edition, John Wiley \& Sons, 2000.

Prakash, R., Colby, L., Flaherty, K. W., Francis, S. R., Guduz, M. E., "Design of a long endurance Titan VTOL System," AIAA Space 2005 Conference, (to be published).

Wertz, James R., and Larson, Wiley J. Space Mission Analysis and Design, Microcosm, 1999.

Honeywell-Garrett 2004 Turbocharger Selection Catalog.

New Frontiers AO, Radioisotope Power Systems (RPS) Summary, October 2003.

NASA General Purpose Heat Source Seminar Slides, 1999. 